\title{
Developing a Process for Returning Medically Actionable Genomic Variants to Latino Patients in a Federally Qualified Health Center
}

\author{
Gabriel Q. Shaibia Iftikhar J. Kullo ${ }^{b}$ Davinder P. Singhe Richard R. Sharp ${ }^{c}$ \\ Eleanna De Filippis ${ }^{f}$ Idali Cuellar ${ }^{c}$ Valentina Hernandez ${ }^{e}$ Sharon Levey ${ }^{c}$ \\ Carmen Radecki Breitkopf ${ }^{c}$ Janet E. Olson ${ }^{c}$ James R. Cerhan ${ }^{c}$ \\ Lawrence J. Mandarino $^{g}$ Stephen N. Thibodeau ${ }^{d} \quad$ Noralane M. Lindor \\ ${ }^{a}$ Center for Health Promotion and Disease Prevention, College of Nursing and Health Innovation, Arizona \\ State University, Phoenix, AZ, USA; ${ }^{b}$ Department of Cardiovascular Sciences, Mayo Clinic, Rochester, MN, USA; \\ 'Department of Health Sciences Research, Mayo Clinic, Rochester, MN, USA; d Department of Laboratory Medicine \\ and Pathology, Mayo Clinic, Rochester, MN, USA; ${ }^{~}$ Mountain Park Health Center, Phoenix, AZ, USA; ${ }^{\mathrm{f}}$ Department of \\ Endocrinology, Mayo Clinic, Scottsdale, AZ, USA; ${ }^{9}$ Division of Endocrinology, Department of Medicine, and Center \\ for Disparities in Diabetes, Obesity, and Metabolism, University of Arizona, Tucson, AZ, USA
}

\section{Keywords}

Precision medicine · Federally Qualified Health Center ·

Return of results - Latino community - Hispanic peoples .

Health disparities

\begin{abstract}
Aim: To develop a process for returning medically actionable genomic variants to Latino patients receiving care in a Federally Qualified Health Center. Methods: Prior to recruitment, researchers met with primary care providers to (1) orient clinicians to the project, (2) establish a process for returning actionable and nonactionable results to participants and providers through the electronic health record, and (3) develop a process for offering clinical decision support for follow-up education and care. A Community Advisory Board was engaged to provide input on recruitment strategies and materials for conveying results to participants. Participants in the Sangre Por Salud (Blood for Health) Biobank with hyperlipidemia or colon polyps represented the pool of potentially eligible participants. Results: A total of 1,621 individuals were invited to participate and 710 agreed to an in-
\end{abstract}

\section{KARGER}

(c) 2018 S. Karger AG, Basel

E-Mail karger@karger.com

www.karger.com/phg person consenting visit (194 no-showed and 16 declined). Over 12-months, 500 participants were enrolled. Participants were primarily Spanish-speaking (81.6\%), female (74.2\%), and enrolled because of hyperlipidemia (95.4\%). All but 2 participants opted to receive primary (i.e., related to enrollment phenotypes) as well as secondary actionable results. Conclusion: Efforts to bring precision medicine to community-based health centers serving minority patients may require multilevel engagement activities to include individuals, providers, health systems, and the community.

(c) 2018 S. Karger AG, Basel

\section{Introduction}

Precision medicine has the potential to improve health and reduce disease burden in individuals based upon their genetic profile. Under ideal conditions, medically actionable genetic findings are linked to evidence-based clinical decision support in order to direct interventions that mitigate or reduce the burden of disease. In this context, medically actionable genetic results may have impli- 
cations across several levels that include patients, family members, providers, health systems, and payers. Similarly, the absence of a medically actionable variant on genetic testing (i.e., negative results) has its own set of implications. Currently, the majority of comprehensive precision medicine efforts in the USA take place in large academic medical institutions and health systems, often implemented within defined research protocols [1].

A limitation of current genomic research is the lack of diversity among participants [2] as well as clinical settings. It is well established that racial and ethnic minorities are underrepresented in clinical research, as are individuals from low-income communities who often lack access to regular primary health care [3]. There is concern that findings from comprehensive precision medicine initiatives, especially psychosocial findings, may not be generalizable to underrepresented minority groups and will be difficult to translate to community health clinics that serve vulnerable populations. If precision medicine initiatives are to have broad applicability and support health equity among vulnerable populations [4], there is a need to include diverse populations and settings in their designs and approaches.

Beginning in 2007, the National Human Genome Research Institute (NHGRI) has sponsored a multicenter consortium called "electronic Medical Records and Genomics" (eMERGE) with a focus on identifying optimal methods to integrate genomic medicine discoveries into clinical practice [5]. Mayo Clinic has been a member of the eMERGE network since its inception. In the most recent phase of funding [6] Mayo Clinic has been collaborating with Mountain Park Health Center (MPHC), a Federally Qualified Health Center (FQHC), to expand precision medicine to a community-based primary care practice. FQHCs are community-based outpatient clinics that qualify for specific federal reimbursement through Medicare and Medicaid to address health disparities through comprehensive primary and preventive care for medically underserved populations [7].

The Arizona portion of Mayo Clinic's eMERGE III project, titled the Arizona Return of Actionable Variants Empirical (RAVE) Study, leveraged the Sangre Por Salud (Blood for Health) Biobank, an existing electronic health record (EHR)-linked biobank of Latino patients from MPHC [8]. The Sangre Por Salud Biobank was designed to facilitate genetic research in an underrepresented Latino community through an FQHC. The purpose of this paper is to describe the rationale, design, procedures, and implementation framework for the Arizona RAVE study at MPHC as part of the larger Mayo Clinic eMERGE III project [9].

\section{Materials and Methods}

\section{Clinical Engagement}

Prior to enrolling participants in 2016, MPHC leadership met with investigators from Mayo Clinic to outline an approach that would engage health care providers and staff in order to develop the processes and guide the implementation of the Arizona RAVE study. The project was presented to primary care providers, behavioral health professionals, and medical assistants during their periodic staff meetings. These opportunities allowed for regular touch points, updates, and feedback from stakeholders at MPHC. Additionally, individuals employed by MPHC were identified to participate in regular research team meetings with investigators to support implementation. A guiding principle for the collaborative project was to find ways to integrate the project into established mechanisms so as not to negatively impact the clinical workflow.

\section{Community Engagement}

In addition to working with MPHC, the research team engaged with the Sangre Por Salud Biobank Community Advisory Board (CAB) on multiple occasions. The CAB was an established entity but had limited exposure to genetic testing projects and return of results. Therefore, initial engagement strategies were educationally oriented to discuss concepts related to actionability, implications of results for individuals and family members, and how to provide follow-up clinical support (medical management and referral). These initial educational discussions laid the groundwork for tangible input from the $\mathrm{CAB}$ on recruitment strategies (e.g., phone scripts and letters) and study-related materials (e.g., consent forms and results letters) as well as their capacity to serve as a sounding board for the potential impact of returning genomic results to participants. Based upon the CAB's feedback, study-related documents and procedures were adapted for cultural, language, and contextual appropriateness. All materials were made available in English and Spanish, and a bilingual/ bicultural research coordinator was hired to recruit and enroll participants. The coordinator worked at MPHC in order to support the day-to-day aspects of the project and to be able to interface directly with MPHC providers, staff, and access health records.

\section{Securing Approvals}

The Mayo Clinic IRB served as the IRB of record for the study and, in accordance with ethical conduct of research involving human subjects, approved the protocol, procedures, and material prior to recruitment. MPHC personnel provided significant input on the research protocol, study-related documents (including review and approval of translations), and procedures. Access to contact information of potentially eligible participants from the Sangre Por Salud Biobank was secured through an established formal application, review, and approval process that takes into account the mission of MPHC and addresses potential implications of the research on their patients and/or the clinical practice. For this particular project, provisions were outlined for uninsured patients who may need follow-up care secondary to the results of genetic testing. Upon IRB approval, a Certificate of Confidentiality was secured through the NHGRI and a working database of potentially eligible participants was created.
Public Health Genomics 2018;21:77-84 DOI: $10.1159 / 000494488$
Shaibi et al. 


\section{Workflow: Recruitment and Enrollment}

Participants identified from the Sangre Por Salud Biobank were invited for an in-person visit with the research coordinator during which the study was introduced and the informed consent process ensued. The decision to implement an in-person visit (as opposed to consenting by mail) included the low response that MPHC historically receives with mail correspondence, the uniqueness of genetic research in this setting, and foreseen challenges with low literacy and poor reading comprehension of written documents. To this point, across the Sangre Por Salud Biobank from which all Arizona RAVE participants were recruited, fewer than $50 \%$ of individuals completed high school [8]. The inperson visit allowed the research coordinator to meet face-to-face with each potential participant, provide a structured educational background on genes and genetic testing using an audiovisual format, explain the study in detail, and answer the participant's questions in real time.

Participants were eligible if they remained active as a Sangre Por Salud Biobank participant and MPHC patient and had dyslipidemia (defined as LDL cholesterol $>140 \mathrm{mg} / \mathrm{dL}$, HDL cholesterol $<40 \mathrm{mg} / \mathrm{dL}$, or triglycerides $>150 \mathrm{mg} / \mathrm{dL}$ ) and/or evidence of colon polyps (any histology). Potentially eligible individuals were sent an invitation letter and/or received a call from the research coordinator. The letter invited participants to call the research coordinator for more information or allowed participants to decline by returning a "Not Interested" notice in a prepaid return envelope. In addition to mailing out letters, the research coordinator made phone calls to potentially eligible participants to explain the project and, if interested, schedule an in-person visit.

During the in-person visit and prior to meeting with the research coordinator, a narrated slide show was used to explain basic concepts related to inheritance and possible risks and benefits of genetic testing. The term "actionable" was also explained. This $\sim 15$-min educational narrative presentation was delivered on a laptop/tablet in the individual's language of choice; it was developed specifically for the Arizona RAVE study population to prepare potential participants for the informed consent discussion (available upon request). The intention of the narrated presentation was to facilitate a more transparent and patient-centered approach to informed decision making regarding participation [10].

The RAVE study offered participants the option of receiving only the results related to familial hypercholesterolemia and colorectal cancer (i.e., "primary results" of a subset of all the sequenced genes) or to receive all of the genetic testing results that were deemed medically actionable, even if not related to the eligibility phenotypes in this study ("secondary results"). Informed consent was obtained by a bilingual/bicultural research coordinator in the participants preferred language. Those participants who provided informed consent went on to complete a survey to evaluate the following broad constructs: (a) understanding of potential genomic results, (b) concerns about having genomic data in the EHR, (c) worries about future employability or insurance coverage, (d) views regarding sharing genomic results with family members and others, (e) perceived challenges and barriers to sharing genomic results with others, (f) intent to pursue medical evaluation or counseling in response to genomic results, and (g) confidence about their decision to pursue genomic testing. Assistance with survey completion was provided as needed. Participants were reimbursed USD 50 for their time.

Returning Actionable Variants

\section{Gene List for Sequencing}

The genes selected for study across the eMERGE III sites included the original 56 genes that were deemed medically actionable by the American College of Medical Genetics (ACMG56) [11]. In addition, each recruitment site proposed additional genes that had varying degrees of medical actionability. The final list of genes and variants agreed upon by consensus among the investigators at eMERGE sites included 109 genes and 1,766 single nucleotide variants. After discussion, the Clinical Actionability Working Group, representing all the eMERGE sites, agreed upon 68 genes and 14 single nucleotide variants as meeting the consensus clinical actionability threshold and quality control indices and were considered for return of results across the eMERGE III sites (see online suppl. Tables 1 and 2; for all online suppl. material, see www.karger.com/doi/10.1159/000494488). Some, but not most genes in the final sequencing panel were related to the Mayo Clinic phenotypes of interest (i.e., hyperlipidemia and/or colorectal neoplasia).

\section{Workflow: Return of Results Process}

Several steps were taken to define the process for returning the genetic results. Per the informed consent document, pathogenic or likely pathogenic variants found in the consensus genes would be returned. It was estimated that $\sim 3 \%$ of participants would have a result of this type. Variants of uncertain significance were not to be returned. For those in whom no pathogenic/likely pathogenic variants were found, the consent form stated they would be provided this finding as well. The iterative process engaged the MPHC Medical Director, the chair of Internal Medicine, the medical providers (physicians and nurse practitioners), the medical assistants, and the Sangre Por Salud CAB in order to outline return of results processes that would be congruent with procedures for returning clinical results within the MPHC practice and acceptable to participants receiving their results. This process is outlined in Figure 1 and includes provisions for returning medically actionable results as well as findings in which no actionable result was found.

\section{Returning Nonactionable (Normal) Results}

A first step towards formalizing the process was to draft a "no actionable results found" letter that would be mailed to participants. This initial draft was presented to the Sangre Por Salud CAB for feedback and suggestions. The $\mathrm{CAB}$ pointed out that this letter may be confusing to participants as they were recruited for a specific risk profile but the genetic testing did not yield an actionable result. Therefore, the $\mathrm{CAB}$ helped craft language to articulate the fact that genes are only one of the many factors that contribute to health and disease. The letter also offered participants the opportunity to speak with a genetic specialist about their nonactionable testing results, if so desired, and encouraged participants to discuss their results with their primary care provider. The revised letter was reviewed and approved by MPHC health care providers and leadership. Due to the aforementioned difficulties with communication by mail in this population, it was decided that participants would also receive a follow-up phone call from the research coordinator to confirm receipt of the letter with an offer to speak with a genetic specialist who could answer any questions about the testing or results. A copy of the results letter along with a copy of the genetic results was placed in the participant's EHR to document what genes had been tested in this study. 


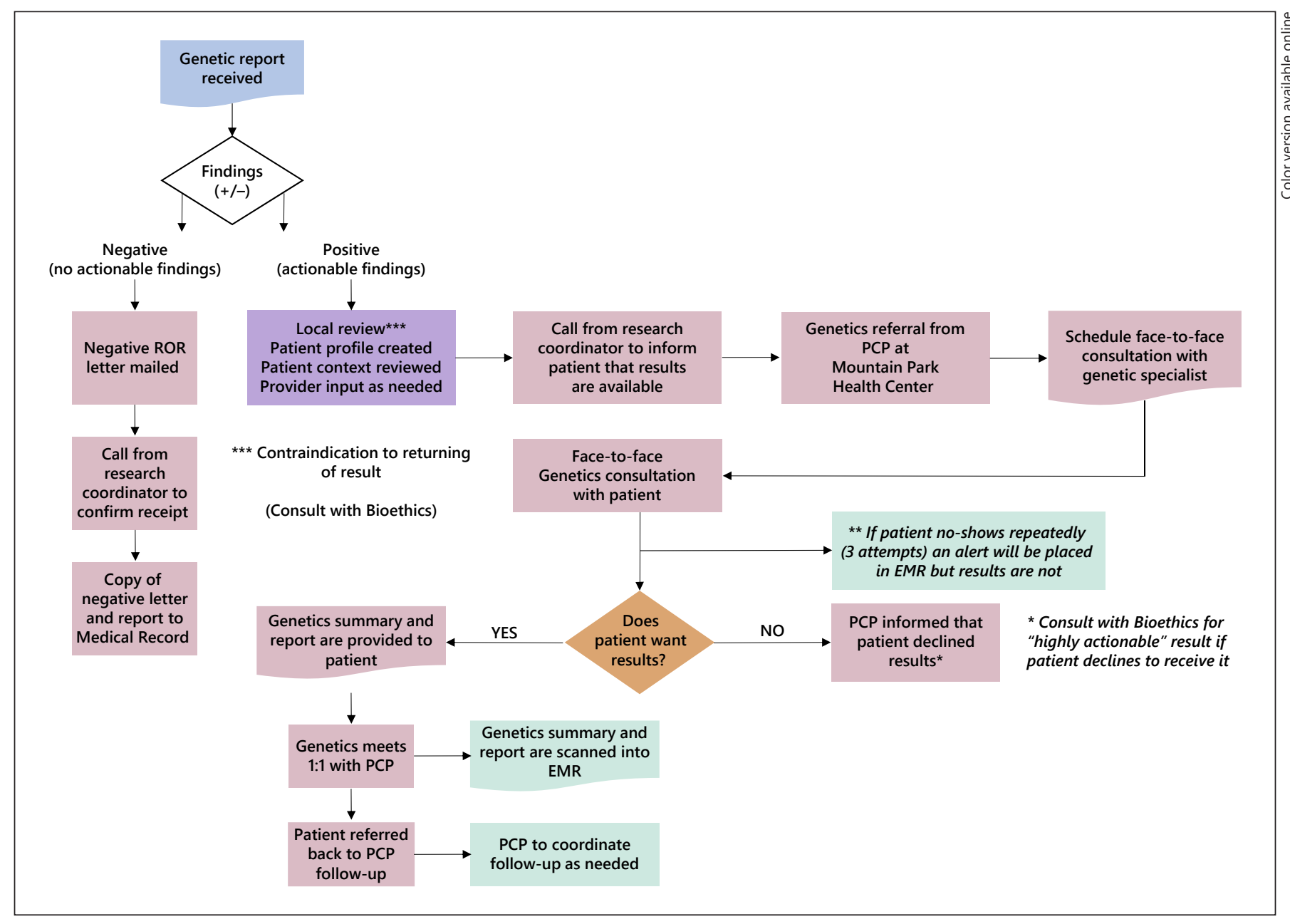

Fig. 1. Flow diagram for returning actionable and non-actionable results.

\section{Returning Actionable Results}

A similar process was followed to outline the steps for returning medically actionable results to participants and providers - i.e., pathogenic or likely pathogenic variants that were detected in a gene and deemed to be medically actionable. The first step in returning these results was to develop a patient profile through a medical chart review to check for potential relative contraindications or complications (e.g., recent suicide attempt, diagnosis of terminal cancer, other) for returning results. If it was determined that there were no contraindications, a referral for genetic counseling (at no charge to the study participant) would be initiated by the patient's MPHC provider followed by a call from the research coordinator to schedule an in-person meeting with a genetic specialist from Mayo Clinic.

A genetic counseling referral by the patient's provider was warranted for several reasons, including to facilitate communication about results and interpretation directly back to the providers through the EHR and to cover the genetic counseling activities from a medicolegal perspective. In the event that a participant could not be reached by phone, a follow-up letter would be mailed to request that patients call the research coordinator. Additionally, an alert would be placed in the EHR so that staff and providers could encourage and support efforts to re-contact the patient. The alert would not disclose specifics of the results but only note that the results were deemed medically actionable. During the results disclosure session, the Mayo Clinic genetic specialist would discuss the results along with potential health implications. Actionable results would not be placed in the EHR until after the results were returned to the patient by the genetic specialist. At the conclusion of the session, participants would be referred back to their provider at MPHC for follow-up management. Concerns were raised at the medical provider meeting regarding the need to have specific, high-quality information provided about the uncommon genetic conditions that might be discovered so that MPHC providers could assist in medical management, as these disorders could be unfamiliar to some providers. To address this concern, the genetic specialist would be available to meet individually with the participant's health care provider to provide information, recommendations, and resources to facilitate management of the patient. Availability of a genetic specialist as a resource was reassuring to providers who would be responsible for ordering any additional tests and coordinating follow-up care if necessary. 


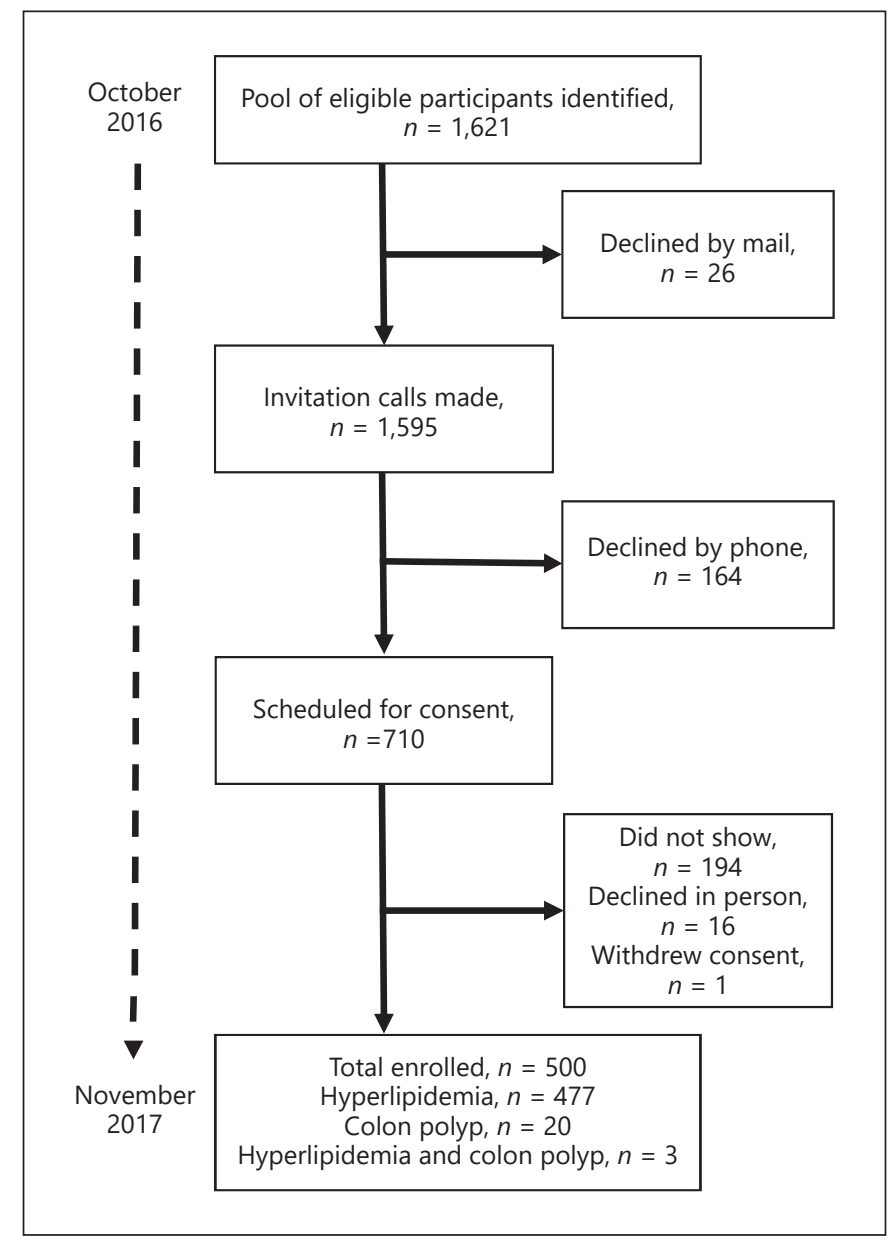

Fig. 2. Recruitment and enrollment of study participants.

\section{Results}

A total of 1,621 participants from the Sangre Por Salud Biobank met the inclusion criteria for the RAVE study. From this list, 26 declined by mail and the research coordinator called the remaining 1,595 individuals. From these potentially eligible participants, 164 declined to participate and 710 agreed to an in-person visit with the coordinator to learn more about the study and sign the consent form if interested in participating. From this group, $194(27.3 \%)$ did not come to their scheduled appointment, $16(3.1 \%)$ declined to participate after hearing more about the study, and 1 withdrew from the study after consenting to participate. In total, the final sample consisted of 500 participants who were enrolled over a 12-month period (Fig. 2).

Compared to the eligible pool of potential participants who did not enroll $(n=1,181)$, those who enrolled $(n=$
Table 1. Descriptive characteristics of the Arizona RAVE population

\begin{tabular}{lcc}
\hline & $\begin{array}{l}\text { English speaking } \\
(n=92)\end{array}$ & $\begin{array}{l}\text { Spanish speaking } \\
(n=408)\end{array}$ \\
\hline Age, years & $43.7 \pm 14.3$ & $48.9 \pm 10.5^{*}$ \\
Male & $22(23.9)$ & $107(26.2)$ \\
Hyperlipidemia & $88(95.6)$ & $389(95.3)$ \\
Colon polyps & $3(3.3)$ & $17(4.2)$ \\
Both & $1(1.1)$ & $2(0.5)$ \\
Consent time, min & $67.0 \pm 16.0$ & $81.0 \pm 20.0^{*}$ \\
\hline
\end{tabular}

Data are presented as $n(\%)$ or mean $\pm \mathrm{SD}$, as appropriate. ${ }^{*} p<$ 0.001 .

500) were older $(45.9 \pm 11.5$ vs. $40.9 \pm 12.5$ years; $p<$ 0.0001 ), more likely to have some form of insurance coverage ( 30.0 vs. $25.4 \% ; p=0.0001)$, more likely to be born outside of the USA ( 84.0 vs. $78.1 \%$; $p<0.01$ ), and less likely to be employed full-time (27.4 vs. $37.2 \% ; p=0.0001$ ). There were no differences in sex, language preference, or educational attainment.

Table 1 describes the demographic characteristics of the cohort, which was primarily Spanish-speaking (81.6\%), female (74.2\%), and enrolled because of dyslipidemia (95.4\%). The low rate of colon polyps reflects both the youth of this cohort and the low rate of routine colonoscopy in the practice. The Spanish-speaking participants were on average 5.2 years older $(p<0.001)$ and required additional time (almost $15 \mathrm{~min}$ ) to complete the consenting process compared to the English-speaking participants $(p<0.001)$. Only 2 participants opted not to receive secondary results (i.e., only genetic results related to hyperlipidemia or colorectal dysplasia) during the informed consent process and the remaining 498 consented to receive both primary and secondary results. The 2 participants who opted not to receive secondary results did not provide any specific rationale for this decision.

\section{Discussion}

It is well documented that Latinos are underrepresented in biomedical research [12] and this disparity may be even greater for genomic medicine studies [13]. A growing body of evidence suggests that limited access to genetic studies is a driving factor underpinning the lack of participation among Latinos and therefore coordinated efforts to support genetic research opportunities for this 
key segment of the population are warranted [14]. Our experiences thus far highlight the myriad practical challenges associated with the pursuit of this important goal.

In addition to enrolling Latino participants and returning sequencing results, a major goal of Arizona RAVE was to establish a process for integrating genomic medicine into an FQHC. To facilitate this aspect of the project, steps were taken to engage clinic providers and staff as well as community members involved in the Sangre Por Salud CAB. We found that early and ongoing dialogue with the providers within the practice was helpful in establishing and refining procedures for how to communicate results and coordinate care in the event that followup testing or referral would be necessary. The approach to formally integrate feedback from providers, staff, and the community was participatory in nature and in line with calls to engage multiple stakeholders to enhance the applicability and overall success of the research [15]. To this end, decisions were made collaboratively and oftentimes through an iterative process across the multiple stakeholders. Although the stakeholders served in an advisory capacity, the research team would defer to their real-world expertise in making decisions as long as they did not violate or compromise the ethical conduct of research involving human subjects.

Through this iterative process, it was decided that patients with actionable results would be informed of their results by a medical geneticist prior to seeing their primary care provider and having the results placed into the medical record. This approach is in line with recent suggestions $[16,17]$ and demonstrated respect for the participant's autonomy, as they could still decide to not learn of these results or opt to not share results with their provider. This process also avoided a scenario in which the primary care provider became aware of an actionable result that the participant him or herself had actively refused to learn or did not yet know about. The primary responsibility for educating participants and their providers about a new genetic diagnosis or risk was assigned to a genetics professional, rather than expecting a busy primary care provider to address patient questions and determine whether and what follow-up was needed. The potential for an informal ethics consult was written into the process in the event of a breakdown in lines of communication that put people at avoidable and unacceptable risks, to consider options further on a case by case basis.

The RAVE study at MPHC strived to understand what may be needed in the future to further expand clinical genomics into primary care. We acknowledge that most FQHCs do not have genetic specialists readily available for providers or patients. Nonetheless, both MPHC and the $\mathrm{CAB}$ have supported these efforts as a way to increase representativeness in precision medicine research and potentially identify unique needs for populations and practices that are outside of traditional academic medical settings. Further, the clinic leadership and CAB are optimistic about the potential for precision medicine initiatives to improve health and prevent disease in the future. Thus, the short-term uncertainty stemming from this research is balanced by future opportunities to address health disparities. The $\mathrm{CAB}$ also encouraged the research team to think about ways to increase awareness of genomic medicine in the community at large through community awareness campaigns, newsletters, community health fairs, and public health forums. To date, these efforts have not materialized beyond initial discussions.

This local support does not minimize the fact that the current shortage of genetic counselors will require that additional mechanisms be established and funded in order to increase access to this expertise for vulnerable and underserved populations. Potential mechanisms to increase the availability of genetic counseling, and specifically those with expertise and interests in community and public health, will likely take a combined approach [18]. To meet the immediate demands, telegenetics is an approach that is well received by patients and could be applied to community settings as a viable option for hardto-reach populations [19]. A recent workforce study completed by the Genetic Counselor Workforce Working Group suggests that, in the near future, the growth in genetic counseling education programs may yield a significant increase in the supply of certified genetic counselors to meet direct patient care needs [20]. As genomic medicine becomes more commonly integrated into clinical practice, physician training programs that prepare medical students and residents to apply genomic medicine in the primary care setting will likely support a more robust approach to support widespread dissemination and implementation of precision medicine across an array of settings and populations [21].

The above efforts could be reinforced by communitybased educational programs to increase awareness of precision medicine with a particular emphasis on low health literacy populations [22]. We relied heavily on the CAB to bring the voice of participants and community members to the table. Discussions with the CAB included soliciting advice on study materials (e.g., recruitment scripts and invitation letters) as well as guidance on how to effectively communicate to participants that actionable results were discovered. For example, the CAB helped develop a
Public Health Genomics 2018;21:77-84 DOI: $10.1159 / 000494488$
Shaibi et al. 
letter for returning results when no actionable results were found (the majority of participants in this study), which would be followed by a phone call from the research coordinator to ensure they received the letter. In contrast, the $\mathrm{CAB}$ felt strongly that participants with actionable results should be contacted first by phone to schedule a visit with the genetic specialist (genetic counselor or a medical geneticist) rather than receive a letter indicating they should call the research coordinator. This suggestion was based on the notion that participants established a relationship with the research coordinator during the consent process and would be more likely to follow through with the appointment if they received a personal invitation by phone rather than a letter in the mail.

A particularly interesting $\mathrm{CAB}$ meeting addressed the issue of possibly detecting a genetic finding that warranted expensive diagnostic interventions to prevent or treat that condition, in an individual with inadequate financial resources. Exploring this topic in small groups, in written surveys and in $\mathrm{CAB}$ discussions, the consensus was that a person's financial situation should not be taken into account when offering a result that might be of major medical significance to that person or their relatives. As genetic results are not yet available for disclosure, it remains to be seen whether this position is upheld under different situations, including those where receipt of results is associated with considerable emotional and financial distress. Of particular interest is whether participation and returning results further contribute to therapeutic misconceptions [23] as this concept may be more readily observed among Spanish-speaking populations [24]. Follow-up surveys and interviews are planned in order to explore/document whether participation in this project resulted in any negative effects on individuals, their families, or the health system.

Several learning opportunities emerged from our early experiences implementing the Arizona RAVE project that may be relevant to others working in similar populations and settings. First, the recruitment process was resourceintensive and protracted; despite making multiple phone calls to schedule and confirm the in-person visit there was a high "no-show" rate. As a reference, the recruitment and enrollment process to reach 500 participants in Arizona was completed over a 12-month period, whereas at the Mayo site in Rochester, MN, 2,500 participants were consented by mail over $\sim 8$ months. Second, despite the development of Spanish language audiovisual educational materials to assist in providing a basic level of understanding, participants still required a substantial amount of time with the research coordinator during the consent process. Common discussions revolved around addressing fears of re- ceiving genetic results, with many participants having a fatalistic outlook (e.g., "I know they will find something") despite the anticipated low percentage of actionable results. Other discussions included how payments may be handled if follow-up was needed, managing expectations, and the idea of leaving information as a legacy to the participants' children and family members. Third, a member of the MPHC staff participated in weekly calls with the research team and was helpful in troubleshooting logistical issues and outlining procedures for integrating return of results processes into the practice. Despite being a large, high-volume practice, there was considerable support and enthusiasm from MPHC leadership as well as the providers for the potential to bring cutting-edge diagnostics to the practice. This enthusiasm was expressed even in the face of the inherent uncertainties associated with this type of project and the potential impact it may have on an already busy practice.

Lastly, regular interactions with the Sangre Por Salud $\mathrm{CAB}$ provided ongoing opportunities to gain insight into how to engage a vulnerable, hard-to-reach population and handle potential issues that may arise from disclosing results of genetic testing. The $\mathrm{CAB}$ challenged the research team to think about the implications of the research beyond the context of study participants or the medical care provided at MPHC, emphasizing the importance of educating the local community about genomics and precision medicine.

The lessons learned during the launch of the Arizona RAVE project will be instrumental for supporting the next phase of the project that focuses on returning genetic results to participants and providing clinical decision support to providers. It is likely that the project will evolve as we receive the sequencing results and report these findings back to participants, providers, and the community at large. Nonetheless, we will follow the approach taken to implement the project and trust that the engagement and communication efforts across multiple stakeholders will continue to inform our collective work to bring precision medicine to a vulnerable and underrepresented population.

\section{Acknowledgments}

This project was supported through the eMERGE Network and funded by the NHGRI through grant U01HG6379 to the Mayo Clinic. Additional support was provided by the Mayo Clinic Center for Individualized Medicine. We thank Dr. Faiz Naioom and the Mountain Park Health Center providers and staff for their support and are indebted to the Sangre Por Salud Biobank Community Advisory Board for their contributions. 


\section{Statement of Ethics}

The study protocol was approved by the Institutional Review Board of the Mayo Clinic and all participants provided written informed consent.

\section{Disclosure Statement}

The authors declare no conflict of interest.

\section{References}

1 Weitzel KW, Alexander M, Bernhardt BA, Calman N, Carey DJ, Cavallari LH, et al.; IGNITE Network. The IGNITE network: a model for genomic medicine implementation and research. BMC Med Genomics. 2016 Jan;9(1): 1 .

2 Popejoy $\mathrm{AB}$, Fullerton SM. Genomics is failing on diversity. Nature. 2016 Oct;538(7624): 161-4.

3 Oh SS, Galanter J, Thakur N, Pino-Yanes M, Barcelo NE, White MJ, et al. Diversity in clinical and biomedical research: a promise yet to be fulfilled. PLoS Med. 2015 Dec;12(12): e1001918.

4 Collins FS, Varmus H. A new initiative on precision medicine. N Engl J Med. 2015 Feb; 372(9):793-5.

5 Gottesman O, Kuivaniemi H, Tromp G, Faucett WA, Li R, Manolio TA, et al.; eMERGE Network. The Electronic Medical Records and Genomics (eMERGE) Network: past, present, and future. Genet Med. 2013 Oct; 15(10):761-71.

6 Fossey R, Kochan D, Winkler E, Pacyna JE, Olson J, Thibodeau S, et al. Ethical considerations related to return of results from $\mathrm{Ge}$ nomic Medicine Projects: the eMERGE Network (Phase III) Experience. J Pers Med. 2018 Jan; $8(1): 8$.

7 Grimaldi P. Latest Medicare benefit: FQHC (Federally Qualified Health Center) services. Healthc Financ Manage. 1993 Jan;47(1):34, 36, 38-40.

8 Shaibi G, Singh D, De Filippis E, Hernandez V, Rosenfeld B, Otu E, et al. The Sangre Por Salud Biobank: facilitating genetic research in an underrepresented Latino community. Public Health Genomics. 2016;19(4):229-38.
9 Kullo IJ, Fan X, Olson J, Jose M, Safarova M, Radecki Breitkopf C, et al. The Return of Actionable Variants Empiric (RAVE) Study, a Mayo Clinic genomic medicine implementation study: design and initial results. Mayo Clin Proc. 2018 Nov;93(11):1600-10.

10 Spatz ES, Krumholz HM, Moulton BW. The new era of informed consent: getting to a reasonable-patient standard through shared decision making. JAMA. 2016 May;315(19): 2063-4.

11 Green RC, Berg JS, Grody WW, Kalia SS, Korf BR, Martin CL, et al.; American College of Medical Genetics and Genomics. ACMG recommendations for reporting of incidental findings in clinical exome and genome sequencing. Genet Med. 2013 Jul;15(7):565-74.

12 McGill N. As Hispanics lag in clinical trials, health researchers take action: Outreach expands. Nations Health. 2013 Sep;43:1-16.

13 Haga SB. Impact of limited population diversity of genome-wide association studies. Genet Med. 2010 Feb;12(2):81-4.

14 Avilés-Santa ML, Heintzman J, Lindberg NM, Guerrero-Preston R, Ramos K, Abraído-Lanza AL, et al. Personalized medicine and Hispanic health: improving health outcomes and reducing health disparities - a National Heart, Lung, and Blood Institute workshop report. BMC Proc. 2017 Oct;11(S11 Suppl 11):11

15 Lavery JV. Building an evidence base for stakeholder engagement. Science. 2018 Aug; 361(6402):554-6.

16 Jarvik GP, Amendola LM, Berg JS, Brothers K, Clayton EW, Chung W, et al.; eMERGE ActROR Committee and CERC Committee; CSER Act-ROR Working Group. Return of genomic results to research participants: the floor, the ceiling, and the choices in between. Am J Hum Genet. 2014 Jun;94(6):818-26.

17 Burke W, Antommaria AH, Bennett R, Botkin J, Clayton EW, Henderson GE, et al. Recommendations for returning genomic incidental findings? We need to talk! Genet Med. 2013 Nov; 15(11):854-9.
18 Stoll K, Kubendran S, Cohen SA. The past, present and future of service delivery in genetic counseling: keeping up in the era of precision medicine. Am J Med Genet C Semin Med Genet. 2018 Mar;178(1):24-37.

19 Hilgart JS, Hayward JA, Coles B, Iredale R. Telegenetics: a systematic review of telemedicine in genetics services. Genet Med. 2012 Sep;14(9):765-76.

20 Hoskovec JM, Bennett RL, Carey ME, DaVanzo JE, Dougherty M, Hahn SE, et al. Projecting the supply and demand for certified genetic counselors: a workforce study. J Genet Couns. 2018 Feb;27(1):16-20.

21 Rubanovich CK, Cheung C, Mandel J, Bloss CS. Physician preparedness for big genomic data: a review of genomic medicine education initiatives in the United States. Hum Mol Genet. 2018 Aug;27(R2):R250-8.

22 Williams JR, Yeh VM, Bruce MA, Szetela C, Ukoli F, Wilkins $\mathrm{CH}$, et al. Precision medicine: familiarity, perceived health drivers, and genetic testing considerations across health literacy levels in a diverse sample. J Genet Couns. 2018 Aug. https://doi.org/10.1007/ s10897-018-0291-z.

23 Halverson CM, Ross LF. Incidental findings of therapeutic misconception in biobankbased research. Genet Med. 2012 Jun;14(6) 611-5.

24 Lakes KD, Vaughan E, Jones M, Burke W, Baker D, Swanson JM. Diverse perceptions of the informed consent process: implications for the recruitment and participation of diverse communities in the National Children's Study. Am J Community Psychol. 2012 Mar; 49(1-2):215-32. 